\title{
Callose plug deposition patterns vary in pollen tubes of Arabidopsis thaliana ecotypes and tomato species
}

\author{
Peng Qin ${ }^{1,2}$, Dylan Ting ${ }^{1}$, Andrew Shieh ${ }^{1}$ and Sheila McCormick ${ }^{1 *}$
}

\begin{abstract}
Background: The pollen grain contains the male gametophyte that extends a pollen tube that grows through female tissues in order to deliver sperm to the embryo sac for double fertilization. Growing pollen tubes form periodic callose plugs that are thought to block off the older parts of the tube and maintain the cytoplasm near the growing tip. The morphology of callose plugs and the patterns of their deposition were previously shown to vary among species, but variation within a species had not been examined. We therefore systematically examined callose plug deposition in Arabidopsis thaliana ecotypes, tested for heritability using reciprocal crosses between ecotypes that had differing deposition patterns, and investigated the relationship between callose plugs and pollen tube growth rate. We also surveyed callose plug deposition patterns in different species of tomato.

Results: We used in vitro grown pollen tubes of 14 different $A$. thaliana ecotypes and measured the distance from the pollen grain pore to the first callose plug (termed first interval). This distance varied among Arabidopsis ecotypes and in some cases even within an ecotype. Pollen tubes without a callose plug were shorter than those with a callose plug, and tubes with a callose plug near the grain were, on average, longer than those with the first callose plug farther from the grain. Variations in the first callose plug position were also observed between different species of tomato.
\end{abstract}

Conclusions: We showed that the position of the first callose plug varied among Arabidopsis ecotypes and in tomato species, and that callose plug deposition patterns were heritable. These findings lay a foundation for mapping genes that regulate callose plug deposition or that determine pollen tube length or growth rate.

Keywords: Callose plugs, Pollen tube growth, Sperm

\section{Background}

In angiosperms, the pollen grain contains the male gametophyte. The male gametophyte extends a pollen tube that grows through female tissues in order to deliver sperm to the embryo sac for double fertilization. Callose, a $\beta$ 1,3-glucan, is the major component of the pollen tube cell wall [1]. Callose can be visualized by staining with decolorized aniline blue [2]. Growing pollen tubes form periodic callose plugs that are thought to block off the older parts of the tube and maintain the cytoplasm near the growing tip [3]. Callose plugs exist in pollen

\footnotetext{
* Correspondence: sheilamc@berkeley.edu

'Plant Gene Expression Center, U.S. Department of Agriculture/Agricultural Research Service and Department of Plant and Microbial Biology, University of California at Berkeley, Albany, CA 94710, USA

Full list of author information is available at the end of the article
}

tubes of all flowering plants, although their morphology and the pattern of callose plug deposition varies among species [4]. Callose plugs were proposed as a critical novelty for accelerated pollen tube growth during angiosperm evolution [5], as angiosperm pollen tubes have callosic walls and callose plugs, whereas callose plugs are absent in pollen tubes of gymnosperms.

Callose plug deposition has been correlated with pollen tube growth rate. For example, the number of callose plugs was used as an indicator of pollen tube growth rate in Hibiscus moscheutos [6,7]. In tomato, antisense lines for the pollen receptor kinase LePRK2 showed abnormal callose plug deposition and slower pollen tube growth than in wild type [8]. LeSHY is a protein that interacts with LePRK2; in Petunia, antisense lines for $S H Y$ showed no callose plugs in pollen tubes,

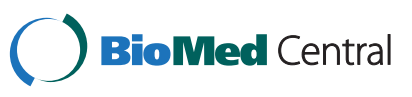


and nearly all the pollen tubes failed to reach ovules [9]. However, in Arabidopsis, one report [10] questioned a need for callose plugs, as pollen tubes carrying a mutant allele of callose synthase 5, cals5-3, had no callose plugs, but grew normally in vitro and in vivo. Seed set on cals5-3 homozygous plants was normal, although transmission of the cal5-3 allele was slightly reduced in heterozygotes (when in competition with WT pollen tubes).

Arabidopsis has been used as a model plant for genetic studies. Many ecotypes have been used to investigate genetic variation in numerous physiological processes [11], and recombinant inbred lines and doubled haploid populations have been used to map genes underlying such variation $[12,13]$. Although callose plug morphology differs in different species [4], to our knowledge variation within a species has not been examined. During the course of other experiments, we noticed that pollen tubes of the Arabidopsis thaliana ecotypes Ler and Columbia ( $\mathrm{Col})$ appeared to vary in the position of the first callose plug; Columbia had the callose plug near the grain, while in Ler the callose plug was farther away. We therefore decided to systematically examine callose plug position in many Arabidopsis ecotypes, and in tomato species. Here we document variation of callose plug deposition in Arabidopsis ecotypes and in tomato species [14] and demonstrate that there is a relationship between callose plug deposition and pollen tube growth.

\section{Results and discussion}

In vitro pollen germination is robust and reproducible in many ecotypes

We first investigated whether the in vitro pollen germination protocol developed in our lab for the Arabidopsis thaliana Columbia ecotype could be used for other ecotypes [15]. For consistency, we always used 2-3 freshly opened flowers for germination assays. After 6 hours germination, we added decolorized aniline blue to visualize callose plugs, transferred the slides to $4^{\circ} \mathrm{C}$ to stop tube growth, and photographed slides on which most pollen grains had germinated and pollen tubes appeared healthy. All but one of the ecotypes tested formed pollen tubes and callose plugs. For measurements, we selected pollen tubes for which it was easy to trace the length of the pollen tube from grain to tip (it was more difficult to carry out such measurements if the pollen tubes were in an area of the slide with many pollen tubes that were tangled together). An unpaired $t$ test showed that the lengths of fifty such randomly selected healthy pollen tubes of each ecotype were not significantly different, in two independent experiments (Figure 1A). We therefore concluded that in vitro pollen germination and tube growth within each ecotype was reproducible. Pollen of the Shahdara ecotype germinated very poorly in this medium, and the few pollen tubes that did form had abnormal morphologies. It is possible that a germination medium suitable for Shahdara could be devised, but we excluded it from further experiments. Although we had initially noticed a difference between the position of callose plugs in Col and Ler, we did not use Ler in these experiments because its germination percentage was considerably lower than in other ecotypes.

\section{The first callose plug position varies among ecotypes in Arabidopsis}

After 6 hours of germination, we measured the first interval length (distance from the pollen grain pore to the first callose plug) of 50 randomly selected pollen tubes in 14 ecotypes. We used the 6 hour time point in order to ensure that all the pollen grains had had ample time to grow a tube, as germination initiation is not synchronous [15]. Within an ecotype, the average lengths of the first interval in two independent experiments were reproducible (Figure 1B) but the average lengths were different in different ecotypes. In about $50 \%$ of the ecotypes, the average length of the first interval was around $50 \mu \mathrm{m}$, indicating that the first callose plug was consistently close to the pollen grain, but in the Ws, C24, Est, and Van ecotypes, the average first interval length was longer, and the standard deviations were extremely large. Large standard deviations were also observed in Cvi, Bay, Nd and Nok.

To further investigate the differences of the first callose plug position in all ecotypes, we pooled together 100 first intervals from two independent experiments and grouped them according to the length of first interval (Figure 1C). There was a wide distribution of first interval lengths among the 14 ecotypes, ranging from $11 \mu \mathrm{m}$ to $394 \mu \mathrm{m}$. We arbitrarily divided pollen tubes into two groups. In the first group the first interval was $<150 \mu \mathrm{m}$, i.e. the first callose plugs were close to the grain; in the second group the first interval was $\geq 150 \mu \mathrm{m}$, i.e. the first callose plugs were farther away from the grain. All ecotypes had some pollen tubes with the first interval $<150 \mu \mathrm{m}$, but some ecotypes also had pollen tubes with the first interval $\geq 150 \mu \mathrm{m}$. The portion of pollen tubes with the first interval $\geq 150 \mu \mathrm{m}$ varied from $0 \%$ (e.g. Col) to 66\% (e.g. Est) among these 14 ecotypes. We further subdivided this group; if the portion of pollen tubes with the first interval $\geq 150 \mu \mathrm{m}$ in one ecotype was lower than $20 \%$, we treated that ecotype as having one pattern of first callose plug deposition, i.e. with the first callose plug close to the pollen grain. However, if the portion of pollen tubes with the first interval $\geq 150 \mu \mathrm{m}$ in one ecotype was higher than $20 \%$, we defined that ecotype as having two deposition patterns. After applying this criterion, Ws, C24, Est and Van had two patterns for first callose plug position (Figure 1C). Thus the first callose plug position not only varied between different ecotypes, but also varied within certain ecotypes. 


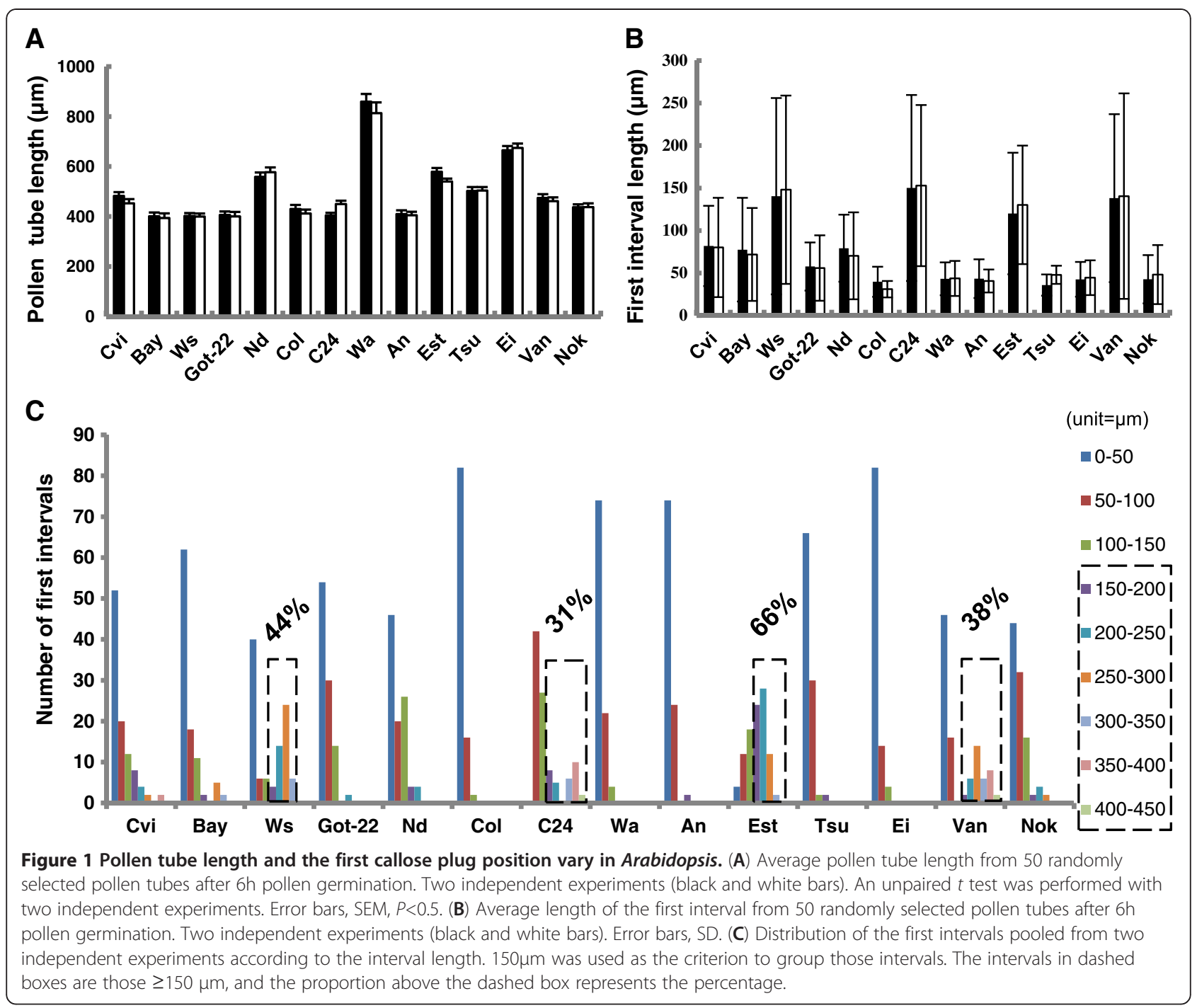

Ecotypes are presumed to be uniform, so this variation of first callose plug position in an ecotype was unexpected. To test whether seed contamination might explain this variation, we used C24. We assayed pollen from 12 individual plants after 6 hours of pollen germination; each showed variation in first callose plug position, and among 12 plants the portions of pollen tubes with the first interval $\geq 150 \mu \mathrm{m}$ ranged from 27 $34 \%$ (Figure 2A), similar to the results shown in Figure $1 C$, suggesting that the variation was not due to seed contamination. We further checked pollen from 6 individual flowers or from individual anthers, each of which still showed variation in the first callose plug position and had similar proportions of pollen tubes with the first interval $\geq 150 \mu \mathrm{m}$ (Figure $2 \mathrm{~B}$ and $\mathrm{C}$ ), indicating that variation of callose plug position in C24 was inherent. Such variation in one ecotype might be due to phenotypic noise $[16,17]$.

\section{Patterns of callose plug deposition are heritable}

To test whether variation of first callose plug position between ecotypes was heritable, we performed reciprocal crosses between ecotypes with different patterns for the first callose plug position. C24 has two patterns while Col has only one pattern (Figure $1 C$ ). The average length of 100 first intervals in the two F1s was intermediate of the parental average lengths (Figure 3A). However, the distributions of the first interval length of the two F1s was similar to that of $\mathrm{C} 24$, and moreover the proportion of pollen tubes of the two F1s with the first intervals $\geq 150 \mu \mathrm{m}$ was larger than $20 \%$ and thus similar to that of C24 (Figure 3B). This indicated that the two F1s had two patterns of first callose plug position, as did C24. This suggested that the pattern of the first callose plug position was heritable and that having two patterns was dominant. The similar first interval lengths in the two F1s indicated that there were no parental effects on 

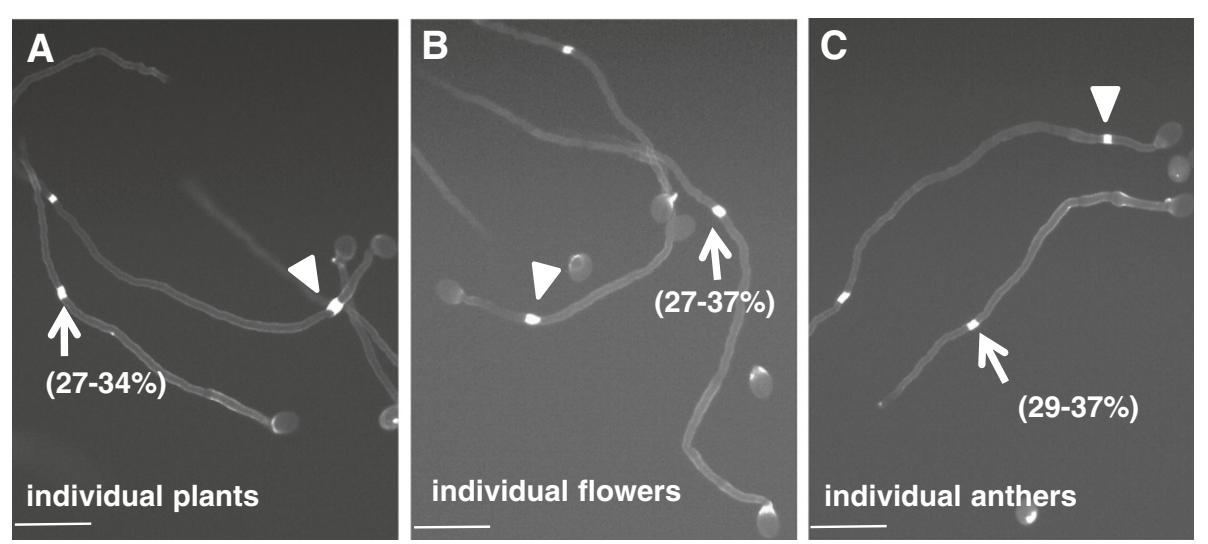

Figure 2 Variation of the first callose plug position from individual C24 plants, flowers and anthers. Variation of the first callose plug in one of 6 individual plants $(\mathbf{A})$, in one of 6 flowers of one plant $(\mathbf{B})$ and one of 6 anthers in one flower $(\mathbf{C})$. The percentages represents the proportion of pollen tubes with the first interval $\geq 150 \mu \mathrm{m}$. Arrows indicate a first callose plug with the first interval $\geq 150 \mu \mathrm{m}$. Arrowheads indicate a first callose plug with the first interval $<150 \mu \mathrm{m}$. Bars $=100 \mu \mathrm{m}$.

callose plug position. Like C24, Ws has two patterns of first callose plug position (Figure 1B). We therefore reciprocally crossed Ws and C24 to investigate how the two patterns of first callose plug position were inherited in the F1s. The average first interval lengths in the two F1s were not significantly different from those of the parents (Figure 3C), moreover, the distributions of the first interval length in the two F1s were also similar to those of the parents. The proportion of pollen tubes with the first interval $\geq 150 \mu \mathrm{m}$ were larger than $20 \%$ (Figure 3D), which indicated that the two F1s had two deposition patterns. We used reciprocal crosses between $\mathrm{Col}$ and An to investigate how the first callose plug position was inherited when the parents only had one pattern for the position of the first callose plug. The distributions of the first interval length in the two F1s were similar to those of the parents (Figure 3F) and no pollen tubes with the first interval $\geq 150 \mu \mathrm{m}$ were observed in either F1, suggesting that these F1s had one deposition pattern, as did the parents. However, the average lengths of the first intervals in the two $\mathrm{Col} \times \mathrm{An}$ F1s were significantly longer than those of the parents (Figure 3E). Together, these results suggested that variation of the first callose plug position in one ecotype was controlled by genetics, and that the trait of two patterns of deposition of the first callose plug was dominant. The dominance was unexpected, as we predicted that callose plug position would be controlled by the gametophyte, as callose plugs are deposited in pollen tubes after pollen germination. Thus in a gametophytic control scenario and assuming that one gene controls the trait, we expected that $50 \%$ of the pollen tubes would have one pattern of first callose plug deposition and 50\% of the pollen tubes would have two patterns. C24 had $31 \%$ pollen tubes that had long first intervals, therefore only $15 \%$ of the F1 pollen tubes were expected to have a long first interval. However, the percentages of pollen tubes with long intervals in F1s from the $\mathrm{Col} \times \mathrm{C} 24$ reciprocal crosses were $35 \%$ and $26 \%$, much more than the expected 15\%. This did not support that this trait was controlled by a single gametophytic gene. If the trait was controlled by multiple genes, a range of intermediate phenotypes between both parental phenotypes might be expected, but that was not the case. Dominance therefore is most consistent with sporophytic control; however, we don't understand how the sporophyte would contribute to this phenotype. Before attempting to genetically map the responsible gene(s), it will be important to consider other parameters that might influence this trait, such as sporophytically-derived pollen wall proteins, or paternal provisioning.

\section{Pollen tube length variability in parents and F1 hybrids}

The average length of the pollen tubes varied in different ecotypes after $6 \mathrm{~h}$ pollen germination (Figure 1A). In most ecotypes, the tube length was around $400 \mu \mathrm{m}$, but in Wa and Ei they were longer, $860 \mu \mathrm{m}$ and $665 \mu \mathrm{m}$, respectively. This implied that Wa and Ei pollen tubes grew much faster than those of other ecotypes. When measuring the patterns of callose plug deposition in parents and F1 hybrids (Figure 3), we also noticed that the pollen tube lengths varied in tested F1 hybrids, but there was no difference in reciprocal crosses (Figure 4A, B and $\mathrm{C})$. The pollen tubes of F1s generated from crosses of $\mathrm{C} 24$ and Col were significantly longer $(618 \mu \mathrm{m}$ and $634 \mu \mathrm{m}$ ) than those of their parents (Col: $430 \mu \mathrm{m}$ and C24: $450 \mu \mathrm{m}$ ) (Figure $4 \mathrm{~A}$ ); this phenomenon was also observed in F1 hybrids from C24 and Ws crosses (Figure 4B), suggesting the possibility of heterosis. However, this possible heterosis was not observed in F1 


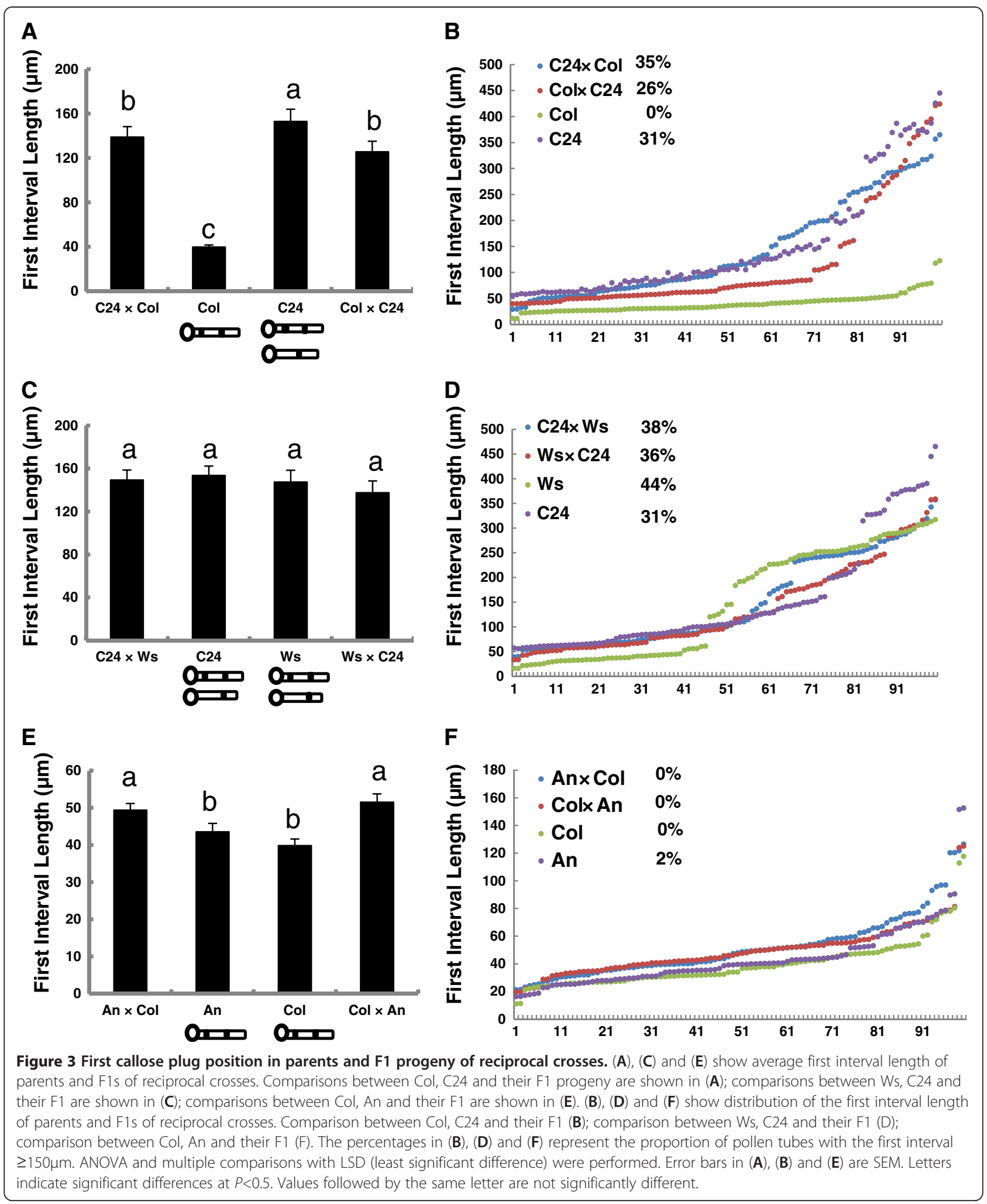

hybrids from crosses of An and Col (Figure 4C). Such differences, i.e. presence or absence of a heterotic effect, have previously been reported, depending on the parents used in the cross [18].
Callose plugs continue to elongate in most ecotypes

We noticed that the length of callose plugs in Col were longer when we had germinated pollen for a long time (30 hours) before staining with aniline blue. Therefore, we 

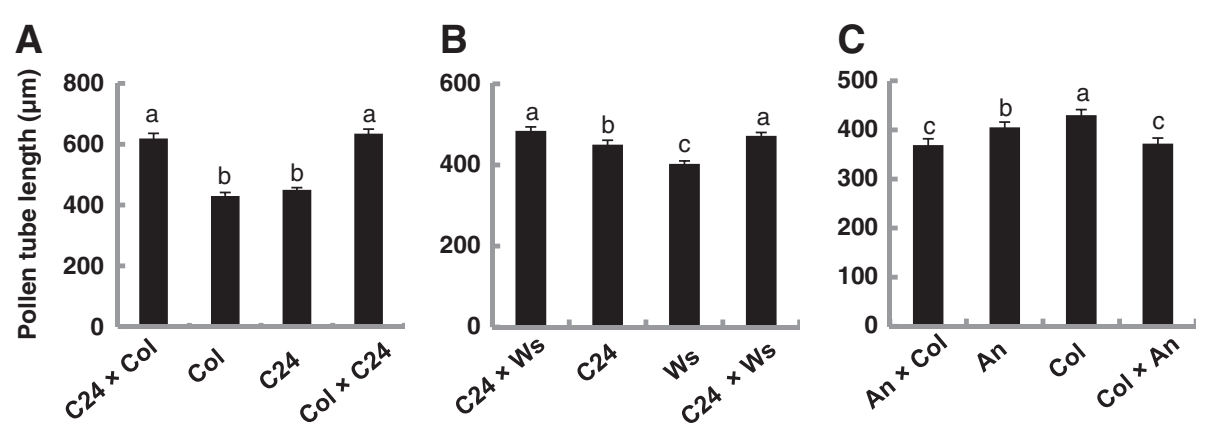

Figure 4 Pollen tube length in parents and F1 progeny. (A), (B) and (C) show average pollen tube length of parents and F1s generated from reciprocal crosses. Comparisons between Col, C24 and their F1 progeny are shown in (A); comparisons between Ws, C24 and their F1 progeny are shown in (B); comparisons between Col, An and their F1 progeny are shown in (C). ANOVA and multiple comparisons with LSD were performed. Error bars are SEM. Letters indicate significant differences at $P<0.5$. Values followed by the same letter are not significantly different.

examined whether this phenomenon occurred in the other ecotypes. After 30 hours of germination, the C24, Van, Bay, Cvi, Ei, Nd, Nok, Got22, Est and Tsu and Ws ecotypes similarly had longer callose plugs (Figure 5B), but the callose plugs of An and Wa were not longer at 30 hours (Figure 5D). In ecotypes with longer callose plugs at 30 hours, it was mostly the first callose plug that had elongated (Figure 5B). To test if this phenotype was heritable, we examined F1 progeny of reciprocal crosses between An and C24. Pollen tubes of the F1s had longer callose plugs after $30 \mathrm{~h}$ germination (Figure $5 \mathrm{E}$ and $\mathrm{F}$ ), suggesting that the C24 phenotype was heritable and dominant. Callose plug elongation implies that callose synthase continues to be active at the callose deposition site, but it is not clear why ecotypes would vary for this parameter.

\section{Callose plug deposition is associated with pollen tube length}

We were interested in determining where and when callose plugs are initiated. However, in the preceding experiments, most callose plugs were completely deposited and we were unable to directly observe callose plug initiation, because aniline blue staining and $4{ }^{\circ} \mathrm{C}$ incubation halts pollen tube growth. We therefore used C24 and a time course experiment (Figure 6A; Table 1) to determine the approximate time that callose plugs started to form. After 2 hours of germination, 34\% of the pollen tubes had a first callose plug close to the grain (first interval $<150 \mu \mathrm{m}$ ), whereas $66 \%$ of the pollen had shorter tubes (Figure 6A)) and had not yet formed a callose plug. After 3 hours of germination, more pollen tubes
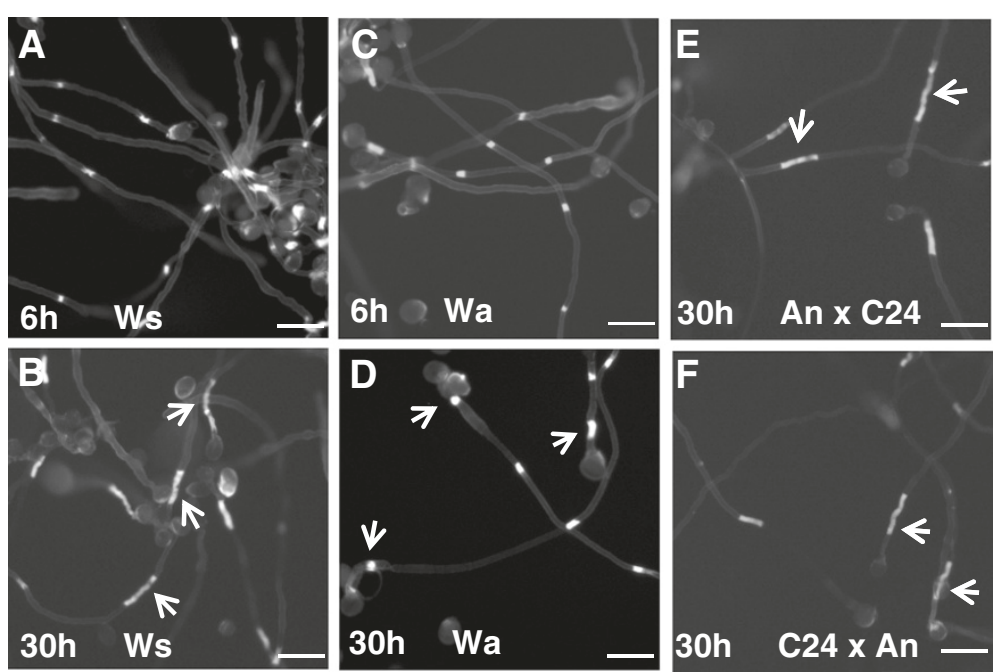

Figure 5 Callose plugs continue to elongate in most ecotypes. Ws pollen tubes after $6 \mathrm{~h}(\mathbf{A})$ or $30 \mathrm{~h}$ (B) germination. Callose plugs of Wa pollen tubes after $6 \mathrm{~h} \mathrm{(C)}$ or $30 \mathrm{~h}(\mathbf{D})$ germination. $(\mathbf{E})$ and $(\mathbf{F})$ Callose plugs of pollen tubes of the F1s from reciprocal crosses between An and C24. Arrows indicate callose plugs. Bars $=50 \mu \mathrm{m}$. 

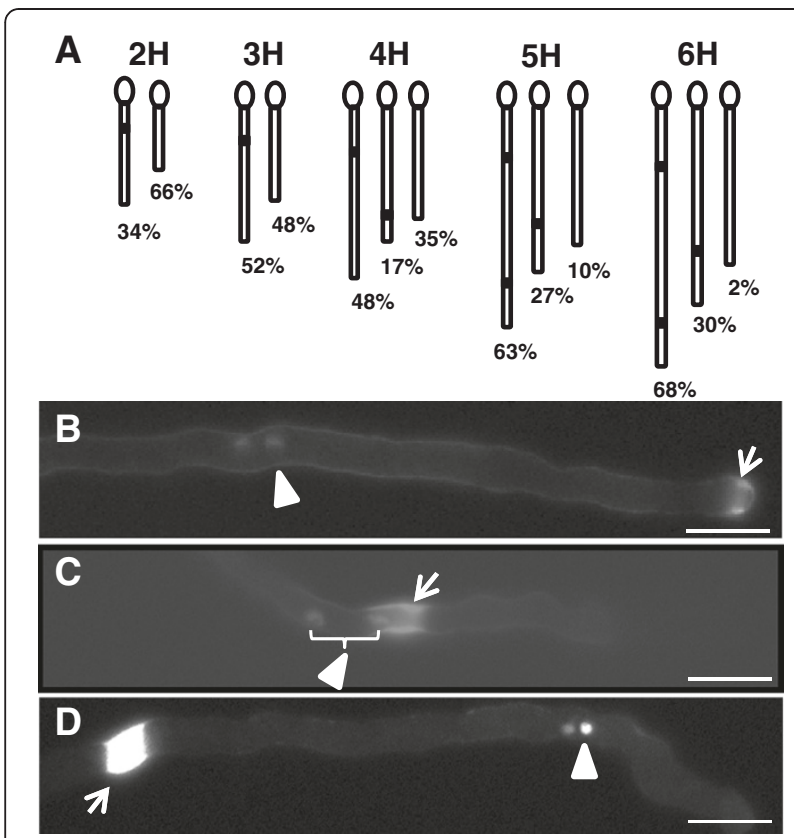

Figure 6 Time course analysis of callose plug formation in the C24 ecotype. (A). Schematic indicating pollen tubes with different callose plug patterns, e.g. the three pollen tubes shown at 4 hours pollen germination represent pollen tubes with a callose plug close to the pollen grain (left), with a callose plug far away from the pollen grain (middle) and one without a callose plug (right). The percentage represents the proportion of pollen tubes with each pattern. Tube lengths in the schematic represent the average pollen tube lengths in Table 1 but are not precisely to scale. (B) A forming callose plug near the pollen tube tip (arrow), sperm cells are behind it (arrowhead). Grain is to the left. (C) Sperm cells (arrowhead) passing through an incomplete callose plug (arrow). Grain is to the left. (D) A completely formed callose plug (arrow), sperm cells are in front (arrowhead). Grain is to the left. Bars $=30 \mu \mathrm{m}$.

had formed a callose plug close to the grain, but about $50 \%$ of the tubes, although longer than at 2 hours, still had no callose plug. After 4 hours of germination, $65 \%$ of the pollen tubes had formed a first callose plug, including $48 \%$ with the first callose plug close to the grain and $17 \%$ with a first callose plug farther away from the grain, suggesting that most of the first callose plugs were formed within 4 hours of germination. Additionally, we noticed that the pattern of callose plugs with the first interval $<150 \mu \mathrm{m}$ shifted to a pattern with the first interval $\geq 150 \mu \mathrm{m}$ between 3 to 4 hours germination, because the percentage of pollen tubes with the first interval $<150 \mu \mathrm{m}$ decreased from $3 \mathrm{~h}$ to $4 \mathrm{~h}$ germination.

To determine where the first callose plugs form, we selected tubes that were forming callose plugs (2-5 hours germination), then determined the position where they started to form. Nearly all (93\%) of the forming callose plugs were found near the pollen tube tip (Figure 6B), indicating that callose plug deposition started near pollen tube tip. This raised a question about the relationship between callose plug position and the position of the sperm cells during pollen tube growth. We therefore used a C24 line in which a $p D U O 1:$ NLS$m R F P$ construct was present, and carried out a time course of pollen germination. The DUO1 promoter is expressed in sperm cells [19] and thus the pDUO1::NLS$m R F P$ reporter can be used to image sperm cell nuclei. The DAPI filter set used to visualize staining with aniline blue overlaps with the mRFP channel, so that we could see both the sperm nuclei and the callose plugs. Some callose plugs (including forming and completely formed callose plugs) were proximal to the position of the sperm (Figure 6B), and some callose plugs were distal to the position of the sperm (Figure 6D). The time course showed that around 30\% of the sperm cells were behind the callose plug at each time point (Table 2). However, in movies generated from the images (e.g., Additional file 1; Figure 6C), sperm cells were seen passing through callose plugs in pollen tubes that had not yet ceased growth after staining with aniline blue. Therefore, it is plausible that the $30 \%$ sperm cells observed behind callose plugs were in tubes in which the aniline blue arrested growth before the sperm cells passed through the callose plug. It was previously reported in tobacco that vegetative and generative cells could be trapped behind callose plugs but could squeeze through a forming callose plug [20].

As many pollen tubes of the C24 ecotype did not have callose plugs after 2 or 3 hours germination, we were able to use C24 to investigate whether callose plug deposition correlated with pollen tube length, without any ecotype effect. The time course analysis (Table 1) was used to compare the lengths of pollen tubes with or without callose plugs. The average tube length of pollen tubes without a callose plug was significantly shorter than those with a callose plug. Additionally, after 4

Table 1 Average length of pollen tubes with and without callose plugs in C24

\begin{tabular}{|c|c|c|c|c|c|}
\hline & $2 \mathrm{~h}$ & 3h & $4 \mathrm{~h}$ & $5 \mathrm{~h}$ & $6 \mathrm{~h}$ \\
\hline tube lengths with callose plug & $242 \pm 11 * *$ & $312 \pm 12^{* *}$ & $391 \pm 11^{* *}$ & $445 \pm 14^{* *}$ & $485 \pm 13^{* *}$ \\
\hline tube length without callose plug & $139 \pm 6$ & $180 \pm 8$ & $234 \pm 8$ & $280 \pm 7$ & $310 \pm 7$ \\
\hline tube lengths with first interval <150um & $242 \pm 11$ & $312 \pm 12$ & $421 \pm 11 *$ & $490 \pm 11^{* *}$ & $533 \pm 17^{* *}$ \\
\hline tube lengths with first interval $\geq 150$ um & & & $362 \pm 11$ & $400 \pm 11$ & $437 \pm 13$ \\
\hline
\end{tabular}

Data are shown with mean $(\mu \mathrm{m}) \pm$ SEM. Significance was determined by an unpaired $t$ test, ${ }^{*} P<0.05,{ }^{*} P<0.01$. 
Table 2 Time course analysis to determine the relationship between sperm cell position and callose plugs

\begin{tabular}{cccc}
\hline Pollen germination hours & A & B & B/A \\
\hline 3 & 27 & 7 & 0.26 \\
4 & 34 & 9 & 0.26 \\
5 & 66 & 23 & 0.35 \\
6 & 128 & 36 & 0.28 \\
\hline
\end{tabular}

A represents the total number of sperm cells investigated.

$B$ represents the number of sperm cells behind the callose plug.

$\mathrm{B} / \mathrm{A}$ represents the proportion of sperm cells behind the callose plug.

hours of germination, the average length of tubes with the first callose plug close to the grain (i.e. first interval $<150 \mu \mathrm{m})$ was significantly longer than tubes with the first callose plug far away from the grain (first interval $\geq 150 \mu \mathrm{m})$. We carried out correlation analyses with the 4, 5 and 6 hour time points; the first interval length and pollen tube length were significantly correlated at 5 hours $(r=-0.235, P<0.05)$ and at 6 hours $(r=-0.242$, $P<0.05)$, but not at 4 hours. Perhaps at the 4 hour time point there was still residual variability due to different times of pollen tube initiation.

\section{Variation of callose plug deposition in tomato species}

To determine if variation in the first callose plug position occurred in other species, we measured the first and second intervals and pollen tube lengths in seven different tomato species. The pollen germination method described in [8] for Solanum lycopersicum worked well for the other six species, and an unpaired $t$ test showed that the average first intervals of 50 randomly selected healthy pollen tubes from two individual experiments were reproducible (Figure 7A). The average length of the first interval varied from $153 \mu \mathrm{m}$ to 363 $\mu \mathrm{m}$ among the 7 species (Figure 7A). The two individual experiments (total of 100 pollen tubes) were pooled and used to determine the distribution of the first callose plug position. The same grouping criterion for first interval length used for Arabidopsis (Figure 1C) was used. Only S. chilense had a large portion (56\%) of pollen tubes with the first interval length $<150 \mu \mathrm{m}$, suggesting that $S$. chilense had two patterns for the first callose plug position. The proportions of pollen tubes with the first interval $<150 \mu \mathrm{m}$ in the other species were smaller than $20 \%$, suggesting that those species mostly had one pattern for the first callose plug position. Although most intervals in those species were grouped into a single pattern, variations were observed (Figure 7B). Thus variation of the first callose position among different species or in one species also exists in tomato. We also noticed that S. pennellii showed two patterns for the second callose plug position: one close to first callose plug (Figure 8A) and another farther away (Figure 8B). We therefore checked all 7 tomato species; all had pollen tubes where the second callose plug position was far away from the first one, but $S$. habrochaites, S. pennellii and S. sitiens also showed a pattern where $22 \%, 50 \%$ or $25 \%$, respectively, of the second callose plugs were near the first one. The phenomenon of callose plug elongation (Figure 5) was not observed in any of the tomato species after prolonged (30 hour) germination.

\section{Conclusions}

Our studies show that all but one of the Arabidopsis ecotypes tested formed pollen tubes during in vitro germination. We showed that the position of the first callose plug varies among Arabidopsis ecotypes and in some cases within one ecotype. The callose plug deposition patterns were heritable; having two patterns of callose plug within an ecotype is dominant. Callose plug deposition correlated with pollen tube length and pollen tube lengths in F1 hybrids sometimes exceeded those of the parents. Variation in callose plug deposition was also seen in species of tomato. These assays were all carried out in vitro, and it is important to acknowledge that there might be differences in the parameters we measured when pollen tubes grow in the pistil, because of potential influences of the female tissue. Although the significance of these callose plug deposition differences are not known, these findings lay a foundation for mapping genes that regulate callose plug deposition or those that determine pollen tube lengths or growth rate.

\section{Methods}

\section{Plant materials and growth conditions}

Fourteen ecotypes of Arabidopsis thaliana (Col-0, Ws-0, C24, Est-1, Shahdara, Van-1, Bay-0, Tsu-1, Cvi-0, Nok-0, Ei-2, An-1, Nd-1, Wa-1, and Got-22) were used. All of the ecotypes except Col-0 were provided by Brian Staskawicz's lab, UC Berkeley; they were originally obtained from NASC and the accession numbers are available there (http://arabidopsis.info/). All Arabidopsis plants were grown in the greenhouse in a 4:1:1 mix of Fafard 4P: perlite: vermiculite under an 18-h light/6-h dark cycle at $21^{\circ} \mathrm{C}$. Flowers of seven tomato species (Solanum lycopersicum, Solanum pimpinellifolium, Solanum chilense, Solanum peruvianum Solanum sitiens, Solanum pennellii, Solanum habrochaites) were obtained from plants grown in UC-Davis greenhouses.

\section{Pollen germination and aniline blue staining}

Arabidopsis in vitro pollen tube germination was carried out as described in [15]. After $6 \mathrm{~h}$ or $30 \mathrm{~h}$ pollen germination, decolorized aniline blue was added and then the slides were immediately transferred to $4^{\circ} \mathrm{C}$. A DAPI 

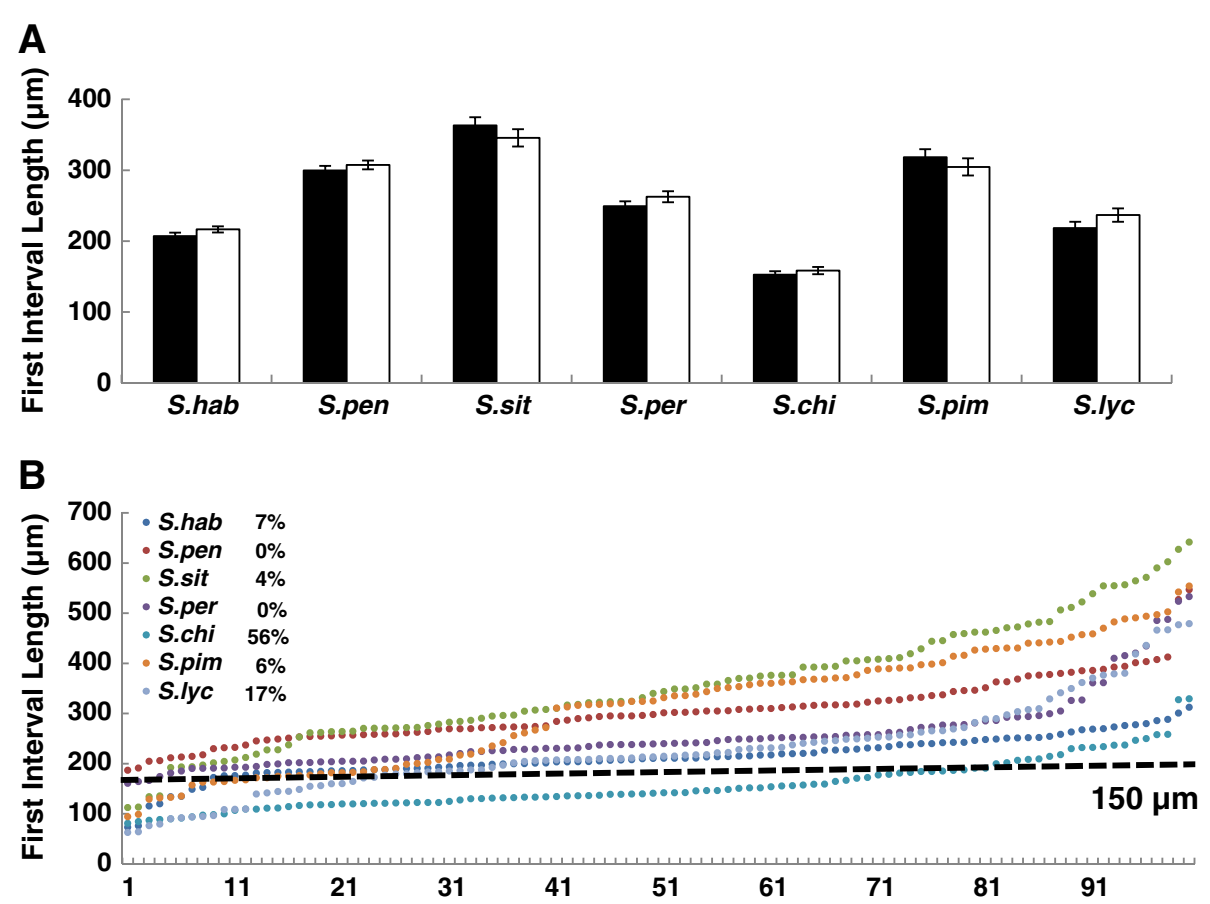

Figure $\mathbf{7}$ Variation in first callose plug position in seven tomato species. (A) Average length of the first interval in two independent experiments (black and white bars). An unpaired $t$ test was performed between two independent experiments. Error bars are SEM, $P<0.5$. (B) Distribution of first interval lengths. $150 \mu \mathrm{m}$ was used as a criterion to group first interval lengths. The percentages represent the proportion of

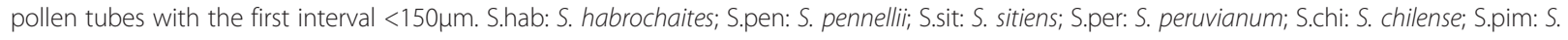
pimpinellifolium; S.lyc: S. lycopersicum.

filter was used to observe callose plug and sperm cells. To observe the movement of sperm cells in the presence of aniline blue, we captured images, using a $40 \times$ objective, immediately after adding decolorized aniline blue (without transferring slides to $4^{\circ} \mathrm{C}$ ). Tomato pollen germination followed the protocol in [8] . Decolorized aniline blue staining was as described in [21].
Microscopic imaging and software for measuring pollen tube lengths and statistical analyses

Microscopic imaging was performed using an Axiovert microscope (Zeiss). Images were captured using a Spot digital camera (Diagnostic Instruments; http://www.diaginc.com/). Distances between a callose plug and the pollen grain pore were measured using Image J software
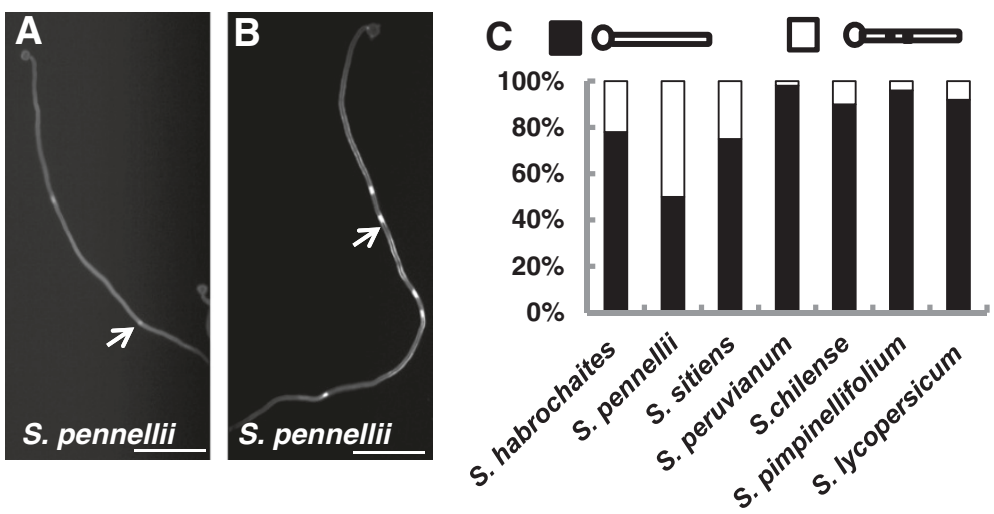

Figure 8 Variation in second callose plug position in tomato species. (A) A pollen tube with the second callose plug (arrow) far away from the first callose plug. (B) A pollen tube with the second callose plug (arrow) close to the first callose plug. (C) The proportions of second callose plug position pattern. Black represents tubes with the second callose plug far away the first callose plug; White represents tubes with the second callose plug close to the first callose plug. Bars in $(\mathbf{A})$ and $(\mathbf{B})=50 \mu \mathrm{m}$. 
(http://rsbweb.nih.gov/ij/) [22]. The unaired $t$ test, correlation analysis, analysis of variance (ANOVA) and multiple comparisons with least significant difference (LSD) were performed using SPSS 10.0 software (SPSS Inc., Chicago, IL, USA).

\section{Additional file}

Additional file 1: Movement of sperm in pollen tube with a

forming callose plug.

\section{Competing interests}

The authors declare that they have no competing interests.

\section{Authors' contributions}

PQ and SM designed the study, PQ, DT and AS performed the experiments, $\mathrm{PQ}$ and SM analyzed the data and wrote the manuscript. All authors read and approved the final manuscript.

\section{Acknowledgements}

We thank Brian Staskawicz's lab for seed of the Arabidopsis ecotypes and Roger T. Chetelat for the flowers from the species of tomato. We thank Binglian Zheng, Guang Wu and Hua Jiang for comments and discussion, and Jorge Muschietti and Weihua Tang for comments on the manuscript. This work was supported by the U.S. Department of Agriculture-Agricultural Research Service Current Research Information System (grant no. 533521000-030-00D). PQ was partially supported by a fellowship from the China Scholarship Council. DT was a participant in the SPUR (sponsored projects for undergraduate research) program of the College of Natural Resources, UC-Berkeley and AS was a participant in UC-Berkeley URAP (undergraduate research apprentice program).

\section{Author details}

${ }^{1}$ Plant Gene Expression Center, U.S. Department of Agriculture/Agricultural Research Service and Department of Plant and Microbial Biology, University of California at Berkeley, Albany, CA 94710, USA. ${ }^{2}$ Current address: Rice Research Institute of Sichuan Agricultural University, Chengdu Wenjiang, Sichuan 611130, China.

Received: 10 May 2012 Accepted: 26 September 2012

Published: 3 October 2012

\section{References}

1. Taylor LP, Hepler PK: Pollen germination and tube growth. Annu Rev Plant Physiol Plant Mol Biol 1997, 48:461-491.

2. Regan SM, Moffatt BA: Cytochemical analysis of pollen development in wildtype Arabidopsis and a male-sterile mutant. Plant Cell 1990, 2(9):877-889.

3. Franklin-Tong VE: Signaling and the modulation of pollen tube growth. Plant Cell 1999, 11(4):727-738.

4. Mogami N, Miyamoto M, Onozuka M, Nakamura N: Comparison of callose plug structure between dicotyledon and monocotyledon pollen germinated in vitro. Grana 2006, 45(4):249-256.

5. Williams $\mathrm{JH}$ : Novelties of the flowering plant pollen tube underlie diversification of a key life history stage. Proc Natl Acad Sci USA 2008, 105(32):11259-11263.

6. Snow AA, Spira TP: Pollen vigour and potential for sexual selection in plants. Nature 1991, 352(29):796-797.

7. Snow AA, Spira TP: Differential pollen tube growth rates and nonrandom fertilization in Hibiscus moscheutos. Amer J Bot 1991, 78(10):1419-1426.

8. Zhang D, Wengier D, Shuai B, Gui CP, Muschietti J, McCormick S, Tang WH: The pollen receptor kinase LePRK2 mediates growth-promoting signals and positively regulates pollen germination and tube growth. Plant Physiol 2008, 148(3):1368-1379.

9. Guyon V, W-h T, Monti MM, Raiola A, Lorenzo GD, McCormick S, Taylor LP: Antisense phenotypes reveal a role for SHY, a pollen-specific leucine-rich repeat protein, in pollen tube frowth. Plant J 2004, 39(4):643-654.
10. Nishikawa S, Zinkl GM, Swanson RJ, Maruyama D, Preuss D: Callose (beta-1,3 glucan) is essential for Arabidopsis pollen wall patterning, but not tube growth. BMC Plant Biol 2005, 5:22-32.

11. Beemster GTS: Variation in growth rate between Arabidopsis ecotypes is correlated with cell division and A-Type cyclin-dependent kinase activity. Plant Physiol 2002, 129(2):854-864.

12. Koornneef M, Alonso-Blanco C, Vreugdenhil D: Naturally occurring genetic variation in Arabidopsis thaliana. Annu Rev Plant Biol 2004, 55:141-172.

13. Seymour DK, Filiault DL, Henry IM, Monson-Miller J, Ravi M, Pang A, Comai L, Chan SWL, Maloof JN: Rapid creation of Arabidopsis doubled haploid lines for quantitative trait locus mapping. Proc Natl Acad Sci USA 2012, 109(11):4227-4232.

14. Peralta IE, Spooner DM, Knapp S: Taxonomy of wild tomatoes and their relatives (Solanum sect. Lycopersicoides, sect. Juglandifolia, sect. Lycopersicon; Solanaceae). Systematic Botany Monographs 2008, vol. 85 American Society of Plant Taxonomists, Ann Arbor, Ml.

15. Boavida LC, McCormick S: Temperature as a determinant factor for increased and reproducible in vitro pollen germination in Arabidopsis thaliana. Plant J 2007, 52(3):570-582.

16. Deplancke B, Verstrepen KJ: Variable outcome of mutations. Science 2012, 335(6064):44-45.

17. El-Samad H, Weissman JS: Noise rules. Nature 2011, 480(7376):188-189.

18. Moore S, Lukens L: An evaluation of Arabidopsis thaliana hybrid traits and their genetic control. G3 (Bethesda) 2011, 1(7):571-579.

19. Rotman N, Durbarry A, Wardle A, Yang WC, Chaboud A, Faure JE, Berger F, Twell D: A novel class of MYB factors controls sperm-cell formation in plants. Curr Biol 2005, 15(3):244-248.

20. Laitiainen $\mathrm{E}$, Nieminen $\mathrm{K}$, Vihinen $\mathrm{H}$, Raudaskoski M: Movement of generative cell and vegetative nucleus in tobacco pollen tubes is dependent on microtubule cytoskeleton but independent of the synthesis of callose plugs. Sexual Plant Reprod 2002, 15(4):195-204.

21. Johnson-Brousseau SA, McCormick S: A compendium of methods useful for characterizing Arabidopsis pollen mutants and gametophyticallyexpressed genes. Plant J 2004, 39(5):761-775.

22. Collins TJ: ImageJ for microscopy. Biotechniques 2007, 43(1 Suppl):25-30.

doi:10.1186/1471-2229-12-178

Cite this article as: Qin et al:: Callose plug deposition patterns vary in pollen tubes of Arabidopsis thaliana ecotypes and tomato species. BMC Plant Biology 2012 12:178.

\section{Submit your next manuscript to BioMed Central and take full advantage of:}

- Convenient online submission

- Thorough peer review

- No space constraints or color figure charges

- Immediate publication on acceptance

- Inclusion in PubMed, CAS, Scopus and Google Scholar

- Research which is freely available for redistribution
C) Biomed Central 